\title{
Projeto de Filtro de Kalman Fuzzy Baseado em Modelo via Decomposição Espectral de Dados Experimentais
}

\author{
Daiana Caroline dos Santos Gomes* \\ Ginalber Luiz de Oliveira Serra** \\ * Universidade Federal do Maranhão, Av. dos Portugueses, 1966, \\ Bacanga, São Luís, Maranhão, (e-mail: daianagomes159@gmail.com). \\ ** Instituto Federal de Educação, Ciência e Tecnologia do Maranhão, \\ Av. Getúlio Vargas, 04, Monte Castelo, São Luís, Maranhão, (e-mail: \\ ginalber@ifma.edu.br)
}

\begin{abstract}
In this paper, a methodology for design of fuzzy Kalman filter (FKF) via spectral decomposition of the experimental data, is proposed. The adopted methodology consists in parametric estimation of local state space linear submodels of a fuzzy model of the dynamic system, by means of a fuzzy algorithm based on least squares, as well as in estimation of FKF gains from the fuzzy model, using the parallel and distributed compensation (PDC) method. The partitioning of experimental data, for definition of the rule base as well as the nonlinear FKF characteristic, is performed by fuzzy C-Means (FCM) clustering algorithm. Considering the PDC method, the Kalman gains in the consequent of each FKF rule are updated as a function of the unobservable components resulting from the spectral decomposition of noisy experimental data. In particular, it allows to FKF efficiency in the face of considerable variations in the noise level over the experimental data (signal/noise ratio). Computational results illustrate the good performance of the proposed methodology when compared to relevant approaches from the literature.

Resumo: Neste artigo é proposta uma metodologia para projeto de filtro de Kalman fuzzy (FKF) via decomposição espectral dos dados experimentais. A metodologia adotada consiste na estimação paramétrica dos submodelos lineares locais de um modelo fuzzy no espaço de estados, referente ao sistema dinâmico, por meio de um algoritmo fuzzy baseado em mínimos quadrados. A estimação dos ganhos do FKF baseado no modelo obtido é realizada pelo método de compensação paralela e distribuída (CPD). O particionamento dos dados experimentais é realizado pelo algoritmo fuzzy $C$-Means (FCM) para definição da base de regras, bem como da característica não linear do FKF. Considerando-se o método CPD, os ganhos de Kalman no consequente de cada regra do FKF são atualizados em função das componentes nãoobserváveis resultantes da decomposição espectral do sinal ruidoso. Este aspecto, em particular, permite a eficiência do FKF diante de variações consideráveis do nível de ruído sobre os dados experimentais (relação sinal/ruído). Resultados computacionais ilustram o bom desempenho da metodologia proposta quando comparada a abordagens relevantes da literatura.
\end{abstract}

Keywords: Systems identification; Fuzzy Kalman filter; Spectral analysis; Parametric estimation; Fuzzy systems.

Palavras-chaves: Identificação de sistemas; Filtro de Kalman fuzzy; Análise espectral; Estimação paramétrica; Sistemas fuzzy.

\section{INTRODUÇÃO}

Em ciências e engenharia, é muito comum a solução de problemas de natureza estocástica como predição, separação e detecção de sinais na presença de ruído (Kasasbeh et al., 2017; Woodbridge et al., 2017; Zhu et al., 2019). O filtro de Kalman (FK) é a ferramenta matemática mais conhecida e utilizada para estimação estocástica a partir de medições ruidosas. Ele foi proposto em 1960 por Rudolph E. Kalman em seu artigo intitulado "A New Approach to Linear Filtering and Prediction Problems", descrevendo uma solução recursiva para o problema da filtragem linear no domí- nio do tempo discreto (Kalman, 1960), tornando-se uma abordagem padrão para estimação ótima. Desde sua publicação, o filtro de Kalman foi objeto de extensa pesquisa e aplicações nas áreas de cálculo de órbita, rastreamento de alvos, navegação integrada, posicionamento dinâmico, fusão de dados de sensor, microeconomia, controle, modelagem, processamento digital de imagem, reconhecimento de padrão, segmentação e detecção de borda de imagem, entre outras. Este amplo interesse pelo FK é devido a sua característica de otimalidade, forma conveniente para processamento online em tempo real, fácil formulação e implementação (Chang et al., 2017; Serra, 2018). 
Nos últimos anos, os sistemas fuzzy vem se destacando em aplicações na área de modelagem e controle de sistemas dinâmicos devido a sua estrutura baseada em regras capaz de fazer aproximação de funções e tratamento de não linearidades e incertezas (Serra, 2012). Alguns estudos envolvendo a utilização de sistemas fuzzy no projeto de filtros de Kalman, já tem sido propostos no intuito de melhorar sua robustez em relação a modelagem de incertezas e perturbações do sistema dinâmico (Pires and Serra, 2019; Eyoh et al., 2018).

A metodologia apresentada neste artigo está baseada na construção de um Filtro de Kalman utilizando modelos fuzzy Takagi-Sugeno (TS) no domínio do tempo discreto. A principal contribuição deste artigo consiste no mecanismo de ajuste dos ganhos de Kalman que compõem a base de regras do filtro de Kalman fuzzy, o qual leva em consideração a decomposição dos estados a serem estimados, corrompidos por ruído, utilizando análise espectral singular.

\section{ESTIMAÇÃO PARAMÉTRICA DO FILTRO DE KALMAN FUZZY}

A classe de modelos fuzzy adotada, para representar o comportamento de um sistema dinâmico não linear com $w$ entradas, $q$ saídas e $n$ variáveis de estado, apresenta a $\left.i\right|^{[i=1,2, \ldots, r]}$-ésima regra, dada por:

$$
\begin{aligned}
& \text { SE } z_{1_{k}} \text { É } M_{i 1} \text { e } \ldots \text { e } z_{p_{k}} \text { É } M_{i q} \\
& \text { ENTÃO }\left\{\begin{array}{l}
\hat{\mathbf{x}}_{k+1}^{i}=\mathbf{A}_{i} \hat{\mathbf{x}}_{k}^{i}+\mathbf{B}_{i} \mathbf{u}_{k} \\
\hat{\mathbf{y}}_{k}^{i}=\mathbf{C}_{i} \hat{\mathbf{x}}_{k}^{i}
\end{array}\right.
\end{aligned}
$$

onde $z_{1_{k}} \ldots z_{p_{k}}$ são as variáveis linguísticas do antecedente, $M_{i q}$ são os respectivos termos linguísticos, $\hat{\mathbf{x}}_{k+1}^{i} \in \mathbb{R}^{n \times 1}$ representa os estados estimados do sistema dinâmico não linear, $\hat{\mathbf{y}}_{k}^{i} \in \mathbb{R}^{q \times 1}$ é o sinal de saída estimada e $\mathbf{u}_{k} \in \mathbb{R}^{w \times 1}$ é o sinal de entrada aplicado no sistema não linear. As matrizes $\mathbf{A}_{i}, \mathbf{B}_{i}, \mathbf{C}_{i}$ são, respectivamente, de estados, entrada e saída, com dimensões $n \times n, n \times w$ e $q \times n$, as quais formam um submodelo linear no espaço de estados que descreve a dinâmica do sistema não linear em torno de um ponto de operação, no consequente da $i$-ésima regra. O modelo fuzzy aproxima o comportamento do sistema dinâmico não linear, através da soma ponderada dos submodelos do consequente, em função do grau de ativação normalizado $\gamma^{i}\left(z_{k}\right)$ da $i$-ésima regra, como segue:

$$
\begin{gathered}
\hat{\mathbf{x}}_{k+1}=\sum_{i=1}^{r} \gamma^{i}\left(z_{k}\right) \mathbf{A}_{i} \hat{\mathbf{x}}_{k}+\sum_{i=1}^{r} \gamma^{i}\left(z_{k}\right) \mathbf{B}_{i} \mathbf{u}_{k} \\
\hat{\mathbf{y}}_{k}=\sum_{i=1}^{r} \gamma^{i}\left(z_{k}\right) \mathbf{C}_{i} \hat{\mathbf{x}}_{k}
\end{gathered}
$$

onde $r$ é o número de regras do modelo fuzzy, tal que:

$$
\sum_{i=1}^{r} \gamma^{i}\left(z_{k}\right)=1, \quad \gamma^{i}\left(z_{k}\right) \geq 0
$$

\subsection{Estimação Paramétrica do Antecedente}

O particionamento dos dados experimentais, de acordo com a metodologia adotada, implicará na definição das regiões de operação e, necessariamente, do número de regras do FKF. Dentre os diversos algoritmos existentes para esta finalidade, o FCM apresenta importante aplicabilidade devido a sua eficiência e simplicidade de implementação (Zhang et al., 2019; Kim et al., 2018). O algoritmo de agrupamento FCM é formulado como segue (Babuska, 1998). Seja um conjunto de dados $\mathbf{Z}$ de tamanho $N$, e a matriz de partição inicial $\mathbf{U}^{(0)}$; escolhe-se o número de agrupamentos $1<r<N$, a tolerância $\epsilon>0$ e o expoente de ponderação $m>1$.

Repetir para $l=1,2, \ldots$

Passo 1- Cálculo dos centros dos agrupamentos:

$$
\mathbf{v}_{i}^{(l)}=\frac{\sum_{k=1}^{N}\left(\mu_{i k}^{(l-1)}\right)^{m} \mathbf{z}_{k}}{\sum_{k=1}^{N}\left(\mu_{i k}^{(l-1)}\right)^{m}}, \quad 1 \leq i \leq r
$$

Passo 2- Cálculo das distâncias:

$$
\begin{gathered}
D_{i k \mathbf{A}}^{2}=\left(\mathbf{z}_{k}-\mathbf{v}_{i}^{(l)}\right)^{T} \mathbf{A}\left(\mathbf{z}_{k}-\mathbf{v}_{i}^{(l)}\right) \\
\text { com } 1 \leq i \leq r, \quad 1 \leq k \leq N \text { e } \mathbf{A}=I
\end{gathered}
$$

Passo 3- Atualização da matriz de partição:

Se $D_{i k \mathbf{A}}>0$ para $1 \leq i \leq r, \quad 1 \leq k \leq N$

$$
\mu_{i k}^{(l)}=\frac{1}{\sum_{j=1}^{r}\left(D_{i k \mathbf{A}} / D_{j k \mathbf{A}}\right)^{2 /(m-1)}}
$$

Senão

$\mu_{i k}^{(l)}=0$ se $D_{i k \mathbf{A}}>0$ e $\mu_{i k}^{(l)} \in[0,1] \operatorname{com} \sum_{i=1}^{r} \mu_{i k}^{(l)}=1$

Até $\left\|\mathbf{U}^{(l)}-\mathbf{U}^{(l-1)}\right\|<\epsilon$

\subsection{Estimação Paramétrica do Consequente}

Uma vez que, de acordo com a metodologia adotada, o projeto do FKF é baseado em modelo, faz-se necessário a estimação das matrizes que compõem o modelo fuzzy, descrito na eq. (1). Para isso, um algoritmo fuzzy baseado em mínimos quadrados, usando-se a abordagem local, foi desenvolvido, onde as partições dos dados experimentais, obtidas pelo algoritmo de agrupamento FCM, serviram como critérios de ponderação para a estimação dos submodelos locais do consequente do modelo fuzzy. 
A equação de estados do modelo fuzzy da eq. (2), para a $i$-ésima regra, pode ser reescrita da seguinte forma:

$\mathbf{x}_{k+1}=\gamma_{k}^{1}\left[\begin{array}{ll}\mathbf{x}_{k}^{T} & \mathbf{u}_{k}^{T}\end{array}\right]\left[\begin{array}{c}\mathbf{A}_{1}^{T} \\ \mathbf{B}_{1}^{T}\end{array}\right]+\cdots+\gamma_{k}^{r}\left[\begin{array}{ll}\mathbf{x}_{k}^{T} & \mathbf{u}_{k}^{T}\end{array}\right]\left[\begin{array}{c}\mathbf{A}_{r}^{T} \\ \mathbf{B}_{r}^{T}\end{array}\right]$

De acordo com a abordagem local, adotada nesta metodologia, e fazendo-se $N$ observações para eq. (8), o $i$-ésimo submodelo é obtido da seguinte forma:

$$
\left[\begin{array}{c}
\mathbf{x}_{1} \\
\mathbf{x}_{2} \\
\vdots \\
\mathbf{x}_{N}
\end{array}\right]=\left[\begin{array}{cccc}
\gamma_{0}^{i} & 0 & \cdots & 0 \\
0 & \gamma_{1}^{i} & \cdots & 0 \\
\vdots & \vdots & \ddots & \vdots \\
0 & 0 & \cdots & \gamma_{N-1}^{i}
\end{array}\right] \mathbf{D}\left[\begin{array}{c}
\mathbf{A}_{i}^{T} \\
\mathbf{B}_{i}^{T}
\end{array}\right]
$$

onde

$$
\mathbf{D}=\left[\begin{array}{cc}
\mathbf{x}_{0} & \mathbf{u}_{0} \\
\mathbf{x}_{1} & \mathbf{u}_{1} \\
\vdots & \vdots \\
\mathbf{x}_{N-1} & \mathbf{u}_{N-1}
\end{array}\right]
$$

Logo, da eq. (9) tem-se que

$$
\mathbf{X}=\boldsymbol{\Gamma}_{i} \mathbf{D}\left[\begin{array}{c}
\mathbf{A}_{i}^{T} \\
\mathbf{B}_{i}^{T}
\end{array}\right]
$$

A eq. (11) pode ser solucionada para cada submodelo correspondente ao consequente das regras fuzzy como segue:

$$
\left[\begin{array}{c}
\mathbf{A}_{i}^{T} \\
\mathbf{B}_{i g}^{T}
\end{array}\right]=\left[\mathbf{D}^{T} \boldsymbol{\Gamma}_{i} \mathbf{D}\right]^{-1} \mathbf{D}^{T} \boldsymbol{\Gamma}_{i} \mathbf{X}
$$

onde

- D é a matriz com os estados em atraso e entrada $\mathbf{u}$ aplicada no sistema dinâmico;

- $\mathbf{X}$ é o vetor de estados em avanço;

- $\mathbf{x}_{k}=\left[\begin{array}{lll}x_{1_{k}} & x_{2_{k}} \ldots x_{n_{k}}\end{array}\right]$ é o vetor com todos os estados do sistema dinâmico;

- $\boldsymbol{\Gamma}_{i}$ é a matriz diagonal de ponderação da $i$-ésima regra fuzzy obtida a partir do algoritmo de agrupamento FCM.

\subsection{Modelo do Filtro de Kalman Fuzzy}

Nesta seção, a formulação da metodologia adotada para a parametrização do Filtro de Kalman Fuzzy baseado em modelo, a partir do processamento dos dados experimentais via análise espectral singular, é apresentado.

Pré-Processamento por Análise Espectral Singular. Seja o conjunto de dados experimentais ruidosos, do sistema dinâmico sob análise, com $N$ amostras, dado por:

$$
\mathbf{Y}=\left[\begin{array}{llll}
y_{1} & y_{2} & \cdots & y_{N}
\end{array}\right]^{T}
$$

A partir dos dados experimentais, uma matriz trajetória $\mathbf{H}$ é definida considerando-se um conjunto de $\rho$ vetores atrasados com dimensão $\delta$, sendo $\delta$ um número inteiro definido pelo usuário, com $2 \leq \delta \leq N-1$ e $\rho=N-\delta+1$, dada por:

$$
\mathbf{H}=\left[\begin{array}{ccccc}
y_{1} & y_{2} & y_{3} & \cdots & y_{\rho} \\
y_{2} & y_{3} & y_{4} & \cdots & y_{\rho+1} \\
y_{3} & y_{4} & y_{5} & \cdots & y_{\rho+2} \\
\vdots & \vdots & \vdots & \ddots & \vdots \\
y_{\delta} & y_{\delta+1} & y_{\delta+2} & \cdots & y_{N}
\end{array}\right]_{\delta \times \rho}
$$

e a matriz de covariância $\mathbf{S}$, é obtida da seguinte forma:

$$
\mathbf{S}=\mathbf{H H}^{T}, \quad \mathbf{S} \in \mathbb{R}^{\delta \times \delta}
$$

Aplicando-se a decomposição em valores singulares à matriz $\mathbf{S}$, obtém-se um conjunto de autovalores em ordem decrescente tal que $\sigma_{1} \geq \sigma_{2} \ldots \geq \sigma_{L} \geq 0$ com seus respectivos autovetores $\phi_{1}, \phi_{2}, \ldots, \phi_{\delta}$.

Considerando-se $d=\max \left\{i\right.$, tal que $\left.\sigma_{i}>0\right\}$ e $\mathbf{V}_{i}=$ $\mathbf{H}^{T} \phi_{i} / \sqrt{\sigma_{i}} \operatorname{com} i=1, \ldots, d$, a decomposição em valores singulares da matriz trajetória $\mathbf{H}$, pode ser reescrita por:

$$
\mathbf{H}=\mathbf{H}_{1}+\mathbf{H}_{2}+\cdots+\mathbf{H}_{d}
$$

onde a matriz $\mathbf{H}_{i}$ é elementar, isto é, apresenta rank igual a 1, e é dada por:

$$
\mathbf{H}_{i}=\sqrt{\sigma_{i}} \phi_{i} \mathbf{V}_{i}^{T}
$$

O reagrupamento das matrizes $\left.\mathbf{H}_{i}\right|^{i=1, \ldots, d}$ em $p$ termos matriciais linearmente independentes $\left.\mathbf{I}_{j}\right|^{j=1, \ldots, p}$, cujos elementos correspondem às $p$ componentes espectrais extraídas, tal que $p \leq d$, resulta em:

$$
\mathbf{H}=\mathbf{I}_{1}+\mathbf{I}_{2}+\ldots+\mathbf{I}_{p}
$$

As $p$ componentes não-observáveis $\left.\alpha^{j}\right|^{j=1, \ldots, p}$ extraídas dos dados experimentais, resultam do procedimento de média diagonal sobre a matriz $\mathbf{I}_{j}$, dado por:

$$
\alpha_{k}^{j}=\left\{\begin{array}{l}
\frac{1}{k} \sum_{\beta=1}^{k+1} I_{j_{\beta, k-\beta+1}} \quad 1 \leq k \leq \delta^{*} \\
\frac{1}{\delta^{*}} \sum_{\beta=1}^{\delta^{*}} I_{j_{\beta, k-\beta+1}} \quad \delta^{*} \leq k \leq \rho^{*} \\
\frac{1}{N-k+1} \sum_{\beta=k-\rho^{*}+1}^{N-\rho^{*}+1} I_{j_{\beta, k-\beta+1}} \quad \rho^{*}<k \leq N
\end{array}\right.
$$

onde $\delta^{*}=\min (\delta, \rho), \rho^{*}=\max (\delta, \rho)$ e $N=\delta+\rho-1$. 
Assim, o FKF apresenta o consequente da $i$-ésima regra, em função da componente espectral não-observável $\alpha^{*}$, dado por:

$$
\begin{aligned}
\hat{\mathbf{x}^{i}{ }_{k}^{-}} & =\mathbf{A}_{i} \hat{\mathbf{x}}_{k-1}^{i}+\mathbf{B}_{i} \mathbf{u}_{k-1} \\
\mathbf{P}_{k}^{i-} & =\mathbf{A}_{i} \mathbf{P}_{k-1}^{i} \mathbf{A}_{i}^{T}+\mathbf{Q} \\
\mathbf{K}_{k}^{i} & =\mathbf{P}_{k}^{i-} \mathbf{C}_{i}^{T}\left[\mathbf{C}_{i} \mathbf{P}_{k}^{i-} \mathbf{C}_{i}^{T}+\mathbf{R}\right]^{-1} \\
\hat{\mathbf{x}}_{k}^{i} & =\hat{\mathbf{x}}^{i}{ }_{k}^{-}+\mathbf{K}_{k}^{i}\left(\alpha_{k}^{*}-\mathbf{C}_{i} \hat{\mathbf{x}}^{i}{ }_{k}\right) \\
\mathbf{P}_{k}^{i} & =\mathbf{P}_{k}^{i-}-\mathbf{K}_{k}^{i} \mathbf{C}_{i} \mathbf{P}_{k}^{i-}
\end{aligned}
$$

onde

$\hat{\mathbf{x}}_{k}^{i}{ }_{k}^{-}$é o estado estimado a priori correspondente ao $i$-ésimo submodelo no instante de tempo $k$;

$\hat{\mathbf{x}}^{i}{ }_{k-1}$ é o estado estimado a posteriori correspondente ao $i$-ésimo submodelo no instante de tempo passado $k-1$;

$\mathbf{P}_{k}^{i-}$ é matriz de covariância do erro a priori;

$\mathbf{P}_{k-1}^{i}$ é a matriz de covariância do erro a posteriori;

$\mathbf{Q}$ é a matriz de covariância do ruído de processo associado a perturbações internas do sistema dinâmico;

$\mathbf{K}_{k}^{i}$ é o ganho de Kalman correspondente ao $i$-ésimo submodelo;

$\mathbf{R}$ é a matriz de covariância do ruído de medição associado a incertezas nas observações do sinal de saída do sistema dinâmico;

$\alpha_{k}^{*}$ é o sinal ruidoso após o pré-processamento por análise espectral singular.

\section{RESULTADOS COMPUTACIONAIS}

Nesta seção, um exemplo computacional de rastreamento dos estados de um sistema dinâmico não linear com comportamento caótico, em ambiente ruidoso, será apresentado. Para ilustrar a eficiência da metodologia proposta, será realizada uma análise comparativa com um Filtro de Kalman Fuzzy baseado na metodologia proposta por (Páramo-Carranza et al., 2017) e largamente citada na literatura, sob o contexto de variação dos níveis de ruído SNR (relação sinal/ruído - do inglês Signal Noise Ratio).

O sistema dinâmico não linear sob análise consiste no atrator caótico de Lorenz, descrito por (Huang et al., 2015):

$$
\begin{aligned}
& \dot{x}_{1}=a\left(x_{2}-x_{1}\right) \\
& \dot{x}_{2}=c x_{1}-x_{2}-x_{1} x_{3} \\
& \dot{x}_{3}=x_{1} x_{2}-b x_{3}
\end{aligned}
$$

onde os parâmetros $a=10, b=8 / 3$ e $c=28$ proporcionam ao sistema não linear um comportamento caótico.

Uma vez que a metodologia proposta para o projeto do FKF é baseada em modelo, faz-se necessário a identificação do sistema não linear supracitado baseado na estrutura de modelo fuzzy adotada conforme eq. (1). Para tal, um conjunto de dados experimentais do sistema dinâmico não linear foi adquirido, de tamanho $N=10000$, a um período de amostragem de $T=1 \mathrm{~ms}$, e o particionamento referente à variável de estado $x_{1}$ foi realizado através do algoritmo de agrupamento FCM para, assim, definir a estrutura do antecedente e o número de regras do FKF. Na implementação do algoritmo de agrupamento FCM, adotou-se a seguinte parametrização: número de agrupamentos $r=3$ e índice de fuzificação $m=1.5$. A partição dos dados experimentais referentes à variável de estado $x_{1}$, obtida pelo algoritmo de agrupamento FCM, serviu como critério de ponderação para a estimação das matrizes que compõem o consequente do modelo fuzzy descrito na eq. (1). Para isso, foi empregada a abordagem local na implementação de um algoritmo fuzzy baseado em mínimos quadrados para o atrator caótico de Lorenz. As matrizes estimadas do modelo fuzzy serviram para o projeto e parametrização do FKF, conforme eq. (20) - (24). O processamento das variáveis de estado, pelo $\mathrm{FKF}$, de acordo com a metodologia proposta, é em função das componentes não observáveis extraídas pela análise espectral singular dos dados experimentais ruidosos do atrator caótico de Lorenz. A dinâmica das variáveis de estado do atrator caótico de Lorenz foram corrompidas por ruído e, variando-se o nível de ruído (SNR), foram obtidas as estimativas dos estados usando-se o FKF baseado na metodologia proposta, através dos resultados de 10 realizações. Nesta aplicação, considerou-se a decomposição dos dados ruidosos referentes à variável de estado $x_{1}$ em 2 componentes espectrais, e usou-se a componente com maior autovalor associado que representasse a dinâmica do atrator caótico de Lorenz. A fim de demonstrar a eficiência do FKF baseado na metodologia proposta, comparada com o FKF proposto por (Páramo-Carranza et al., 2017), utilizou-se o critério de validação MSE (Mean Square Error) como índice de avaliação de desempenho no processo de estimação dos estados do atrator caótico de Lorenz. Os valores de MSE, para diferentes níveis de ruído (SNR), considerandose o efeito de 10 realizações na estimação das variáveis de estado $x_{1}, x_{2}$ e $x_{3}$ são mostrados nas Figuras (1)(3), respectivamente. Observa-se que, com a metodologia

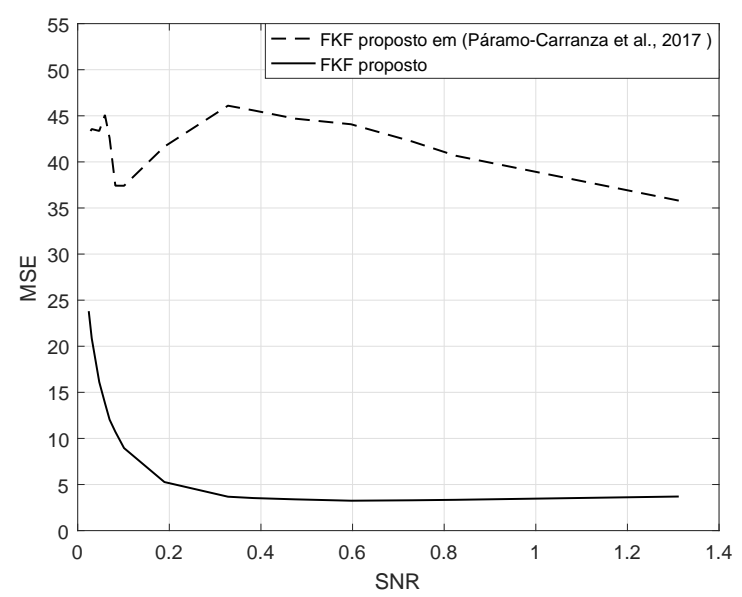

Figura 1. Valores de MSE resultantes do processo de estimação da variável de estado $x_{1}$, considerando-se o efeito de 10 realizações.

proposta neste artigo, a dinâmica das variáveis de estado do atrator caótico de Lorenz foram rastreadas de forma eficiente, apresentando um desempenho superior quando comparado à metodologia proposta em (Páramo-Carranza et al., 2017), para diferentes níveis de ruído. Considerandose o caso particular de $S N R=0,65\left(Q=1 \times 10^{-4} \mathrm{e}\right.$ 


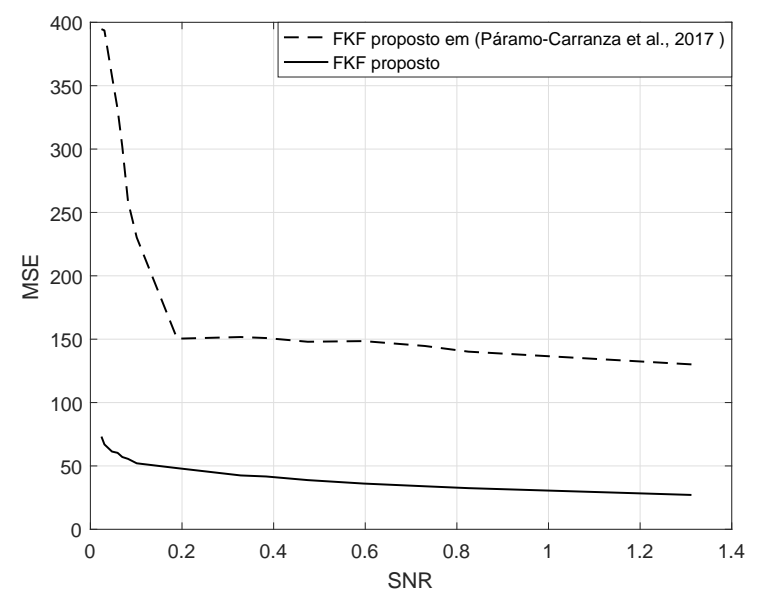

Figura 2. Valores de MSE resultantes do processo de estimação da variável de estado $x_{2}$, considerando-se o efeito de 10 realizações.

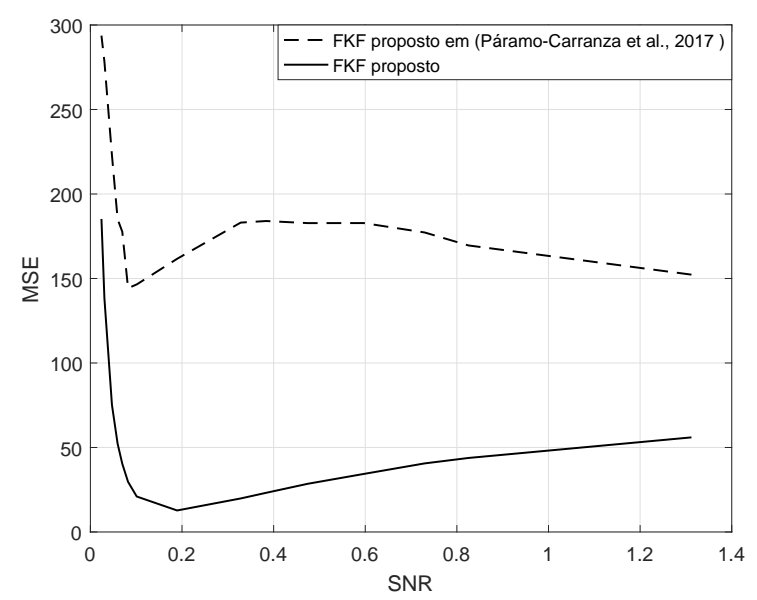

Figura 3. Valores de MSE resultantes do processo de estimação da variável de estado $x_{3}$, considerando-se o efeito de 10 realizações.

$R=100$ ), o comportamento referente à variável de estado $x_{1}$, do atrator caótico de Lorenz, bem como as componentes espectrais extraídas, são mostrados nas Figuras 4-6. Uma vez que a componente $\alpha^{2}$, mostrada na Figura 6 , está mais correlacionada à dinâmica da variável de estado $x_{1}$, enquanto que a componente $\alpha^{1}$, mostrada na Figura 5 , apresenta um comportamento mais correlacionado ao ruído, a componente $\alpha^{2}$ foi utilizada para implementação do FKF proposto, no processo de estimação das variáveis de estado do atrator caótico de Lorenz. As estimações das variáveis de estado $x_{1}, x_{2}$ e $x_{3}$, do atrator caótico de Lorenz, usando-se a metodologia proposta neste artigo, estão ilustradas na Figura 7. As estimações das variáveis de estado $x_{1}, x_{2}$ e $x_{3}$, do atrator caótico de Lorenz, usandose a metodologia proposta por (Páramo-Carranza et al., 2017), estão ilustradas na Figura 8. Verifica-se, portanto, a eficiência e o melhor desempenho do FKF proposto neste artigo.

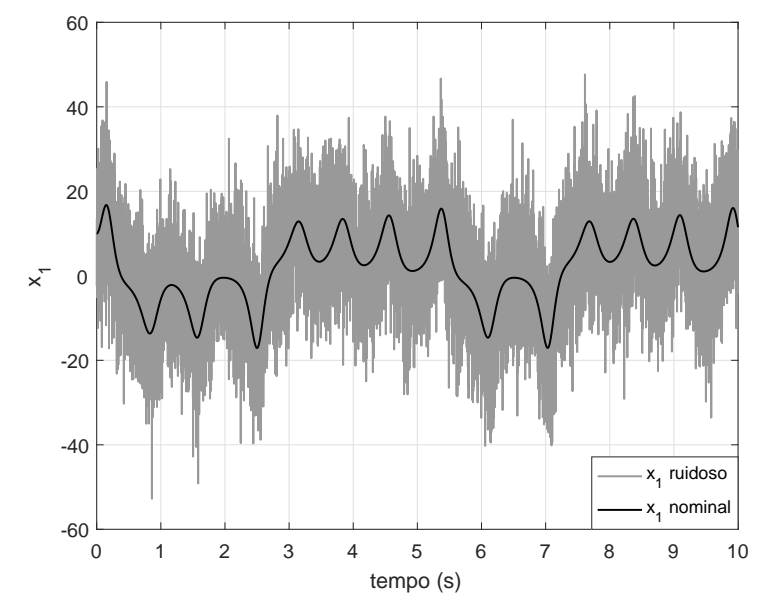

Figura 4. Variável de estado $x_{1}$ do atrator caótico de Lorenz corrompida por ruído com $S N R=0,65$.

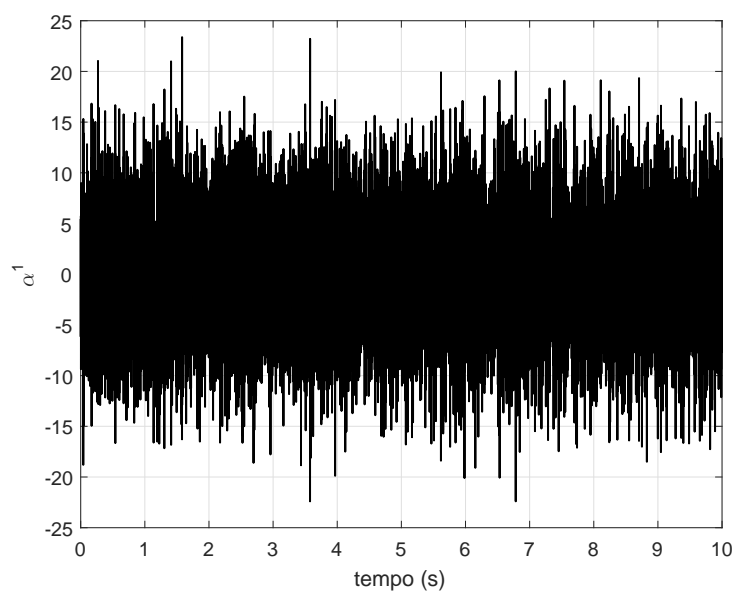

Figura 5. Componente espectral $\alpha^{1}$ extraída da variável de estado $x_{1}$ em ambiente ruidoso com $S N R=0,65$.

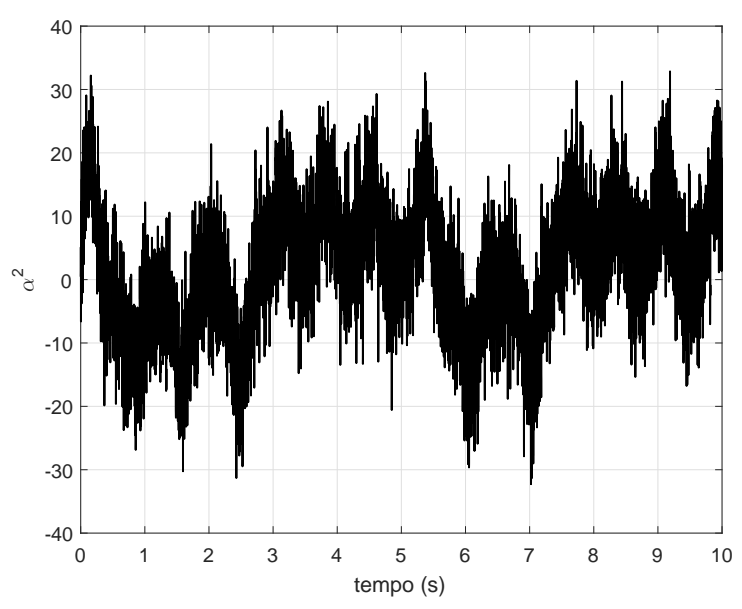

Figura 6. Componente espectral $\alpha^{2}$ extraída da variável de estado $x_{1}$ em ambiente ruidoso com $S N R=0,65$.

\section{CONCLUSÕES}

Neste artigo, o projeto de um Filtro de Kalman Fuzzy baseado em modelo, em função das componentes espectrais 

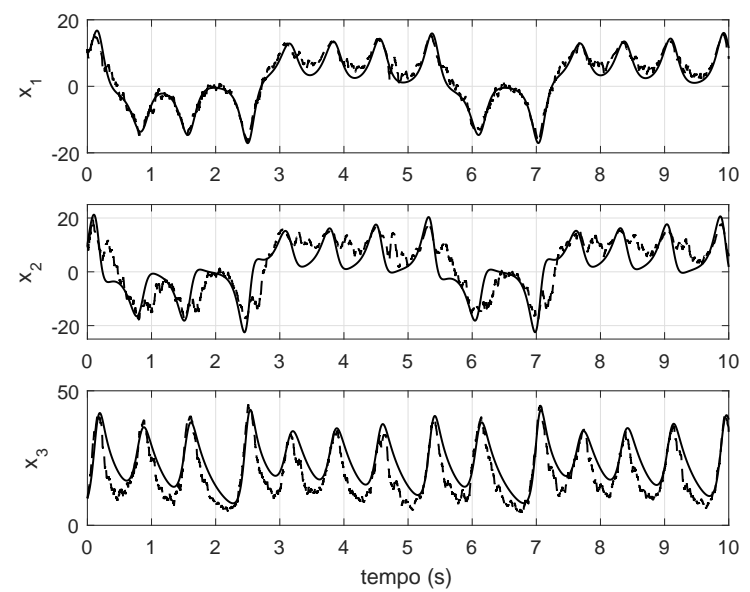

Figura 7. Estimações das variáveis de estado $x_{1}, x_{2}$ e $x_{3}$ pelo FKF baseado na metodologia proposta neste artigo (linha tracejada), comparadas aos comportamentos nominais das respectivas variáveis (linha sólida), para o caso de $\mathrm{SNR}=0.65$.
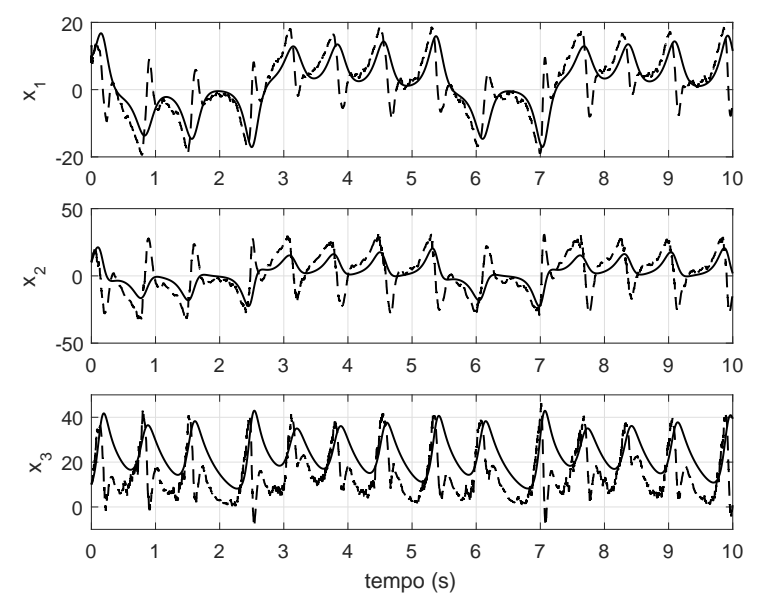

Figura 8. Estimações das variáveis de estado $x_{1}, x_{2}$ e $x_{3}$ pelo FKF baseado na metodologia proposta em Páramo-Carranza et al. (2017) (linha tracejada), comparadas aos comportamentos nominais das respectivas variáveis (linha sólida), para o caso de $\mathrm{SNR}=$ 0.65 .

dos dados experimentais, foi proposto. A partir dos resultados obtidos, o FKF baseado na metodologia proposta mostrou-se eficiente na filtragem e estimação dos estados de um sistema dinâmico não linear com comportamento caótico. A covariância dos ruídos de processo e de medição são variáveis empíricas e foram definidas de acordo com o trabalho utilizado para fins de comparação. O número de componentes a serem extraídas dos dados do sistema dinâmico pode ser alterado dependendo da necessidade do projetista. Para fins de trabalhos futuros, a formulação e aplicabilidade desta metodologia no contexto multivariável com modelos fuzzy tipo-2, é de particular interesse.

\section{AGRADECIMENTOS}

Os autores agradecem à CAPES pelo apoio financeiro, e ao Programa de Pós-Graduação em Engenharia de
Eletricidade da Universidade Federal do Maranhão (PPGEE/UFMA) pelo suporte ao desenvolvimento desta pesquisa.

\section{REFERÊNCIAS}

Babuska, R. (1998). Fuzzy Modeling for Control. International Series in Intelligent Technologies. Kluwer Academic Publishers.

Chang, G., Xu, T., and Wang, Q. (2017). Alternative framework for the iterated unscented kalman filter. IET Signal Processing, 11(3), 258-264.

Eyoh, I., John, R., Maere, G.D., and Kayacan, E. (2018). Hybrid learning for interval type-2 intuitionistic fuzzy logic systems as applied to identification and prediction problems. IEEE Transactions on Fuzzy Systems, 26(5), $2672-2685$.

Huang, Y., Zhang, P., and Zhao, W. (2015). Novel grid multiwing butterfly chaotic attractors and their circuit design. IEEE Transactions on Circuits and Systems II: Express Briefs, 62(5), 496-500. doi:10.1109/tcsii.2014. 2385274 .

Kalman, R.E. (1960). A new approach to linear filtering and prediction problems. Transaction of the ASME, 82 .

Kasasbeh, H., Viswanathan, R., and Cao, L. (2017). Noise correlation effect on detection: Signals in equicorrelated or autoregressive(1) gaussian. IEEE Signal Processing Letters, 24(7), 1078 - 1082.

Kim, E.H., Oh, S.K., and Pedrycz, W. (2018). Design of reinforced interval type-2 fuzzy c-means-based fuzzy classifier. IEEE Transactions on Fuzzy Systems, 26(5), $3054-3068$.

Páramo-Carranza, L.A., Meda-Campaña, J.A., de Jesús Rubio, J., Tapia-Herrera, R., Curtidor-López, A.V., Grande-Meza, A., and Cázares-Ramírez, I. (2017). Discrete-time kalman filter for takagi-sugeno fuzzy models. Evolving Systems, 8(3), 211-219. doi:10.1007/ s12530-017-9181-0.

Pires, D.S. and Serra, G.L.O. (2019). Methodology for evolving fuzzy kalman filter identification. International Journal of Control, Automation and Systems, 17(3), 793-800.

Serra, G.L.O. (ed.) (2012). Frontiers in Advanced Control Systems. Intech.

Serra, G.L.O. (ed.) (2018). Kalman Filters - Theory for Advanced Applications. InTech. doi:10.5772/intechopen. 68249.

Woodbridge, Y., Elidan, G., and Wiesel, A. (2017). Signal detection in complex structured para normal noise. IEEE Transactions on Signal Processing, 65(9), 2306 2316.

Zhang, Y., Bai, X., Fan, R., and Wang, Z. (2019). Deviation-sparse fuzzy c-means with neighbor information constraint. IEEE Transactions on Fuzzy Systems, 27(1), 185-199. doi:10.1109/tfuzz.2018.2883033.

Zhu, X., Wang, T., Bao, Y., Hu, F., and Li, S. (2019). Signal detection in generalized gaussian distribution noise with nakagami fading channel. IEEE Access, 7(9), $23120-23126$. 